\title{
Low-temperature structure of magnetite studied using resonant x-ray scattering
}

\author{
Y. Joly, ${ }^{1}$ J. E. Lorenzo, ${ }^{1}$ E. Nazarenko, ${ }^{2}$ J.-L. Hodeau, ${ }^{1}$ D. Mannix,${ }^{1,3}$ and C. Marin ${ }^{4}$ \\ ${ }^{1}$ Institut Néel, CNRS and Université Joseph Fourier, BP 166, F-38042 Grenoble Cedex 9, France \\ ${ }^{2}$ Institute of Physics, South Federal University, Rostov-on-Don 344090, Russia \\ ${ }^{3}$ XMaS CRG, European Synchrotron Radiation Facility, F-38043 Grenoble Cedex, France \\ ${ }^{4}$ DRFMC/SPSMS/Groupe Matériaux, CEA, F-38054 Grenoble, France
}

(Received 1 August 2008; revised manuscript received 11 September 2008; published 29 October 2008)

\begin{abstract}
We propose a model for the $\mathrm{Fe}$ atomic displacements in the low-temperature phase of magnetite $\left(\mathrm{Fe}_{3} \mathrm{O}_{4}\right)$, based on the analysis of the photon energy dependence of the scattered intensity of selected reflections in a resonant x-ray scattering experiment. The symmetry of the displacement pattern is forced to be consistent with the $C c$ space group, long time claimed to be the actual symmetry of the low-temperature phase. Fe positions at octahedral sites and the corresponding charges are accounted for by a fitting procedure comparing simulations and experiment. We found a pattern of small distortions in the $a-b$ plane. An independent sensitivity to the charge occupancy permits to refine the model of charge ordering previously proposed. Finally we have computed the electric moment of the combined charge displacements to be $1.5 \mu \mathrm{C} / \mathrm{cm}^{2}$.
\end{abstract}

DOI: 10.1103/PhysRevB.78.134110

PACS number(s): 61.05.cc, 71.30.+h

\section{INTRODUCTION}

Magnetite $\left(\mathrm{Fe}_{3} \mathrm{O}_{4}\right)$ is the archetype compound of a number of physical properties and applications. An accurate description of this compound is thus mandatory for the understanding of its own properties as well as a reference for similar effects in related materials. Particularly, the magnetic and magnetoelectric properties of magnetite exhibit an anomaly at $T_{V}=121 \mathrm{~K}$ that still defies complete understanding. ${ }^{1}$ First suggested by Verwey ${ }^{2}$ and expected in many oxides, ${ }^{3,4}$ the low-temperature phase transition in magnetite has been associated to a charge disproportion on the metal atom sites. Theoretical predictions ${ }^{5,6}$ have recently supported Verwey's scheme of the localization of the charge on a more complex pattern, and nuclear magnetic resonance ${ }^{7}$ and Mössbauer ${ }^{8}$ experiments are compatible with different oxidation states of the octahedral iron sites. In a recent paper we have directly confirmed the occurrence of charge ordering ${ }^{9}$ using resonant $\mathrm{x}$-ray scattering (RXS) at the $\mathrm{K}$ edge of iron. The results of our work are in agreement with the Goff et al. ${ }^{10}$ analysis but are apparently in contradiction with the Subías and García et al. papers. ${ }^{11,12}$ In this last case the supposed contradiction was more a matter of terminology (When the charge ordering is not with the formal, ionic charges $2+/ 3+$ can we really speak of charge ordering?) and sensitivity (the Bragg reflections measured in their paper are hardly sensitive to the charge ordering found by us) than a problem of validity. Strictly speaking when two sites are not symmetry equivalent, the corresponding charges cannot be exactly equal. Since then RXS investigations at the $L_{2,3} \mathrm{Fe}$ edges ${ }^{13}$ or at the $\mathrm{K}$ oxygen edge ${ }^{14}$ have confirmed our result. At this edge the photoelectrons probe the $d$ orbitals which are supposed to be charge but also orbitally ordered. In these experiments, the charge ordering occurrence reposes on the measurement of the symmetry breaking $\left(0,0, \frac{1}{2}\right)$ reflection, the sole diffraction peak available at these low photon energies. The origin of the Verwey transition remains a matter of debate as it can be seen in the large number of contributions that have recently appeared in the literature ${ }^{15-19}$ and any progress in the structure and charge determination of this canonical compound is welcomed.

The determination of the atomic positions issued from the metal-insulator transition challenges the scientific community ever since Verwey's seminal work. Magnetite still remains as a difficult problem for conventional crystallography and the low-temperature low symmetry complete structure is not perfectly known yet. The use of traditional x-ray or neutron-diffraction crystallography is especially challenging due to the enormous number of parameters to be accounted for. The actual best refinement has been recently performed in the Pmca space group by Wright et al. ${ }^{20}$ in a combined $\mathrm{x}$-ray and neutron powder diffraction work. Complexity is greatly reduced in this structure model, where there are six nonequivalent iron atoms, two in tetrahedral sites and four in octahedral sites $\left(\mathrm{Fe}_{1}, \mathrm{Fe}_{2}, \mathrm{Fe}_{3}\right.$ and $\left.\mathrm{Fe}_{4}\right)$.

In this paper we go a step further toward the complete resolution of the geometric and electronic configuration of the low-temperature structure of magnetite. We noticed in our previous paper that among the iron octahedral sites, the so-called $\mathrm{Fe}_{1}$ and $\mathrm{Fe}_{2}$ sites are especially sensitive to the magnitude of the charge ordering parameter. On the contrary the uncertainty on the $\mathrm{Fe}_{3}$ and $\mathrm{Fe}_{4}$ iron sites remained important and, as we concluded, more investigation is necessary to refine these sites. The uncertainty follows from the choice to analyze the data within the Pmca space group, as has been done in conventional crystallography. ${ }^{20}$ In addition we have observed peaks indexed on a $(2 \times 2)$ superstructure in the $(a, b)$ plane, a proof that Pmca space group needs to be revisited and that a larger unit cell has to be introduced. Indeed detailed neutron-diffraction studies have proposed $C c$ symmetry in this supercell. This space group is also compatible with the occurrence of an electric moment found by Miyamoto and Shindo ${ }^{21}$ for which a noncentrosymmetric space group is required.

The goal of this paper is thus to provide a successful attempt to solve this structure in the $C c$ space group. A key feature of our work is the simultaneous refinement of structure as well as of the charge disproportion. This paper is organized as follows. In Sec. II we present a brief summary 
of the theory, and of the necessary tools to solve such a problem. Then the new experiments showing the different reflections measured around the iron $\mathrm{K}$ edge are presented. In Secs. VI and VII we explain the strategy to fit the different parameters and we give the results we have been able to reach for magnetite.

\section{RESONANT X-RAY SCATTERING AS A TOOL TO SOLVE A STRUCTURE}

The atomic scattering factors become complex at energies around the absorption edge of the resonant atom in a given material. ${ }^{22}$ As a consequence, diffraction near the edges violates Friedel's law $[I(\vec{Q}) \neq I(-\vec{Q})]$ and can be used, for instance, to identify enantiomorphs. Moreover it permits to highlight a specific atom from the rest of the atoms in a given structure, which is especially useful when the crystallized molecule contains a large number of atoms. ${ }^{23}$ It is also useful to stress the contrast between elements of close atomic number. The multiple-wavelength anomalous diffraction (MAD) is a technique where complete sets of diffraction peaks are collected at a number of photon energies around the resonant(s) atom and simultaneously refined. This method is extensively used in the crystallography of biological materials, and especially of proteins. Our technique, RXS, is different. We record the energy dependence of the intensity of many diffracted peaks in a relatively small energy range around the edge where the atomic scattering amplitude is highly sensitive to the electric and geometrical local environment of the absorbing atoms. In the specialized literature this technique is also called diffraction anomalous near edge structure (DANES).

\section{A. Theory}

Resonant X-ray scattering is a technique where both the power of site selective diffraction and the power of local absorption spectroscopy regarding atomic species are combined to the best. ${ }^{24}$ Reflections are recorded over some tens of $\mathrm{eV}$ around the absorption edge of the elements present in the material, where they show strong energy and angular dependencies. This phenomenon is due to the virtual photon absorption-emission associated with the resonant transition of an electron from a core level to some intermediate state above the Fermi level. By virtue of the dependence on the core level state energy and the three-dimensional electronic structure of the intermediate state, this technique is especially suited to study charge, ${ }^{25,26}$ orbital $^{27,28}$ or spin orderings $^{29}$ and associated geometrical distortions. ${ }^{30,31}$

Core resonant spectroscopies depend on the transition matrix elements of matter-radiation interaction:

$$
M_{n g, j}^{i(o)}=\left\langle\psi_{n}\left|\hat{O}_{i(o)}\right| \psi_{g, j}\right\rangle,
$$

where $\psi_{g, j}$ and $\psi_{n}$ are, respectively, the core ground state centered around the $j$ th atom and the photoexcited state. In the $\mathrm{x}$-ray range, the operator $\hat{O}$ is written through the multipolar expansion of the photon field up to electric dipole (E1) and quadrupole (E2) terms:

$$
\hat{O}_{i(o)}=\vec{\epsilon}_{i(o)} \cdot \vec{r}\left(1+\frac{1}{2} i \vec{k}_{i(o)} \cdot \vec{r}\right),
$$

where $\vec{r}$ is the electron position measured from the absorbing ion, $\vec{\epsilon}_{i(o)}$ is the polarization of the incoming (outgoing) photon, and $\vec{k}_{i(o)}$ is its corresponding wave vector. The sign in front of the imaginary part reveals the use of the negative time arrow. The resonant term, $f_{j}^{\prime}+i f_{j}^{\prime \prime}=f_{j}$, entering in the total atomic structure factor is related to these previous equations. Its complex conjugate is given by the following expression: ${ }^{32}$

$$
f_{j}^{*}(\omega)=-\frac{m_{e}}{\hbar^{2}} \sum_{n, g} \frac{\left(E_{n}-E_{g, j}\right)^{2} M_{n g, j}^{o *} M_{n g, j}^{i}}{\hbar \omega-\left(E_{n}-E_{g, j}\right)+i \frac{\Gamma_{n}}{2}},
$$

where $\hbar \omega$ is the photon energy, $m_{e}$ is the electron mass, $E_{g, j}$ is the ground-state energy, $E_{n}$ is the energy of the excited states, and $\Gamma_{n}$ is the sum of the inverse lifetime of the excited states and of the core hole. The sum in the equation is extended over all the excited states of the system. The complex conjugate permits to make the link between the physical convention where the time arrow is negative and the usual convention in crystallography where $f^{\prime}(\omega)$ is negative, $f^{\prime \prime}(\omega)$ is positive, and the time arrow is positive. When the incoming and outgoing polarizations are parallel, $f^{\prime \prime}(\omega)$ is proportional to $\mu(\omega) \times \hbar \omega$, where $\mu(\omega)$ is the absorption cross section.

In RXS the global process of photon absorption, virtual photoelectron excitation, and photon re-emission is coherent throughout the crystal, thus the incident and diffracted $x$-ray beams give rise to the usual Bragg diffraction condition. Including the anomalous (or resonant) part, the structure factor is written as

$$
F(\vec{Q}, \omega)=\sum_{j} e^{i \vec{Q} \cdot \vec{R}_{j}}\left[f_{0 j}+f_{j}^{\prime}(\omega)+i f_{j}^{\prime \prime}(\omega)\right] .
$$

$\vec{R}_{j}$ stands for the position of the scattering ion $j, \vec{Q}$ is the diffraction vector, and $f_{0}$ is the usual Thomson factor.

We can notice from Eq. (4) that geometrical parameters (the positions of the iron atoms) influence the spectra in two ways. The first one is in the energy dependence of the atomic (but embedded in its neighborhood) scattering factor, $f_{j}(\omega)$, and the second one is in the Bragg phase factor as in conventional x-ray diffraction.

By its dependency on the incident and outgoing polarizations and wave vectors, the anomalous scattering amplitude becomes highly anisotropic. This phenomenon is usually described using either Cartesian or spherical tensor algebra. The advantage of the latter set is that each tensor component can be related to a physical feature of the resonant specie, for example, the magnetic moment, the electric moment and so on. ${ }^{33}$ The best manifestation of this anisotropy is the occurrence of scattering at the so-called forbidden Bragg peaks, for example, the $(0,0,2),(0,0,6)$ or $(-4,4,2)$ reflections in the $F d \overline{3} m$ magnetite. The intensity of these peaks is nearly extinct off-resonance by the nonsymmorphic elements of the space group, glide planes and/or screw axis. In this study we found that the shape of the spectra is only weakly dependent on these angles (but their global intensity yes). For this rea- 


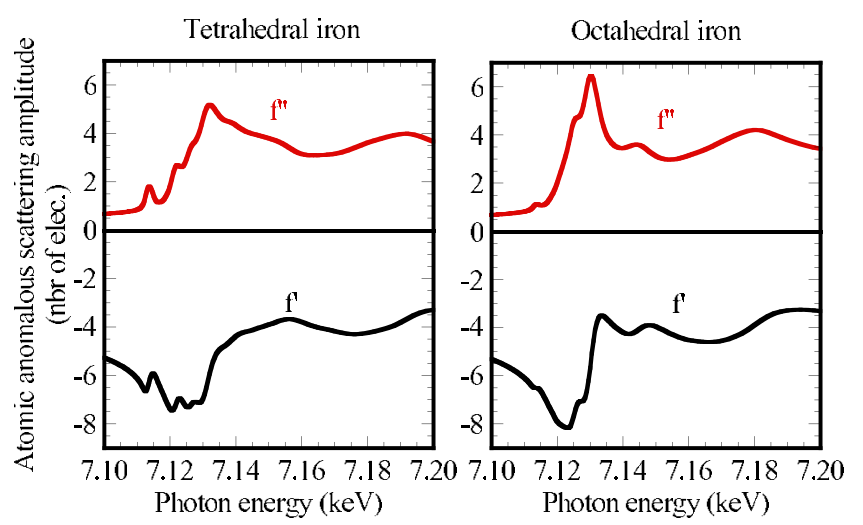

FIG. 1. (Color online) Calculated energy variation of the isotropic part of the E1-E1 atomic anomalous scattering amplitude for the iron in magnetite, top is $f^{\prime \prime}$ and down $f^{\prime}$. On the left the tetrahedral site is shown and the octahedral one on the right. Calculations have been performed for a $7 \AA$ cluster. Shapes are very different.

son we do not further develop this aspect which is nevertheless present in the simulations; no approximation has been used on this anisotropy.

\section{B. Sensitivity to the charge ordering}

Equation (4) explains the sensitivity of resonant diffraction to charge ordering. Indeed atoms with closely related site symmetries but with barely different charges exhibit resonances at slightly different energies. For some reflections the Bragg factor makes the atomic structure factor from these slightly different atoms nearly subtract. Such reflections, usually weak in intensity, are consequently sensitive to tiny differences between the charge state of the resonant specie. In addition, the influence of the often associated geometrical distortions has to be disentangled from pure charge effect.

The $a b$ initio calculation of the resonant part of the structure factor [given in Eq. (4)] is a delicate task. This is especially true when multielectronic phenomena govern the electronic properties of the material, for example, the charge disproportion or when there is a strong interaction between the core-hole and the intermediate states. A RXS experiment at the $\mathrm{K}$ edge covers a range of about $100 \mathrm{eV}$ around the resonance and therefore calculations have to be performed in this broad energy range. The structures appearing just above the Fermi level (also called preedge features) are rich in information but sometimes difficult to simulate due to the presence of correlated electrons. At the absorption K edges, $f_{j}(\omega)$ are dominated by the main E1-E1 (dipole-dipole) contribution probing the empty $p$ states and not by the so-called preedge features and therefore the contribution of correlated electrons can be discarded in the calculations. This assumption is not valid at the $L$ edges and the $a b$ initio procedure should include the multiplet effects and the electron correlations.

The shape of these edges is a signature of the geometrical structure around the absorbing atoms. To illustrate this we show in Fig. 1 typical atomic anomalous scattering amplitudes for the $\mathrm{Fe}$ in magnetite in two different symmetries, tetrahedral and octahedral. They have different shapes and the main edge is not exactly at the same energy. The stronger preedge at $7.115 \mathrm{keV}$ for the tetrahedral site is a signature of the lack of inversion center symmetry at these sites.

The valence state of the resonant atom is seen through the energy shift of its main absorption edge. The energy shift, $\Delta$, of the core state $\left[E_{g, j}\right.$ in Eq. (3)] is directly related to the variation of the atomic electronic occupancy rate, $\delta$, with respect to a nominal value. In the case of magnetite we already noted ${ }^{9}$ that a $\Delta=+0.8 \mathrm{eV}$ shift corresponds to $\delta$ $=-0.1$ electron difference and this relation is roughly linear. Such linear relation is valid because the local octahedral structure is hardly modified through the phase transition. Once summed over the different sites, this simple shift of the atomic scattering factors can give rise to specific features in the spectra which are a signature of charge disproportion. Suppose a phase transition giving, for each site $j$, a displacement $\delta \vec{R}_{j}$ and a shift $\Delta_{j}$. A new peak resulting from this transition can be expressed as

$$
F(\vec{Q}, \omega)=\sum_{j} e^{i \vec{Q} \cdot\left(\vec{R}_{j}+\delta \vec{R}_{j}\right)}\left[f_{0 j}+f_{j}\left(\hbar \omega+\Delta_{j}\right)\right] .
$$

Using a Taylor expansion in $\delta \vec{R}_{j}$ and $\Delta_{j}$ and keeping only the first order, one gets

$$
F(\vec{Q}, \omega) \approx \sum_{j} e^{i \vec{Q} \cdot \vec{R}_{j}} \times\left\{i \vec{Q} \cdot \delta \vec{R}_{j}\left[f_{0 j}+f_{j}(\omega)\right]+\frac{\partial f_{j}(\omega)}{\partial \omega} \Delta_{j}\right\} .
$$

The zero order of the expansion, not shown here, corresponds to the Bragg reflections of the underlying undistorted structure. This equation has two terms. The first one is related to the atomic displacements. The second term is proportional to the energy shift (and thus to the number of electrons). It is responsible for the "charge ordering features" of the Bragg reflections and is proportional to the derivative of the scattering factor. Peaks sensitive to charge disproportion will be such that the atoms of the same charge (same sign in $\Delta_{j}$ ) are out of phase with respect to the atoms with a different charge. Most often both terms contribute to the structure factor, sometimes they add, sometimes they subtract. The importance of a given Bragg reflection to charge ordering is directly related to the weight of the first term with respect to the second. The maximum of this derivative is going to be at the resonance edge, more than $8 \mathrm{eV}$ above the Fermi level and a monoelectronic calculation generally suffices. The price to pay is that calculations have to be carried out on a large cluster because the final states are highly not localized.

Perhaps the most striking example that evidences the power of RXS on analyzing charge structures is the understanding of the charge order in the low-temperature phase of $\mathrm{NaV}_{2} \mathrm{O}_{5} \cdot{ }^{34}$ The phase transition occurring at $T_{C}=34 \mathrm{~K}$ makes the high-temperature unit cell (containing a single $\mathrm{V}$, with charge +4.5) become expanded in a $2 a \times 2 b \times 4 c$ with up to $64 \mathrm{~V}$ atoms. By using the second term in Eq. (6) alone, the charge ordering part, and with the help of only three reflections, the whole charge ordering pattern and domains have been uncovered. Refinement of the charge ordered has been undertaken in different charge ordering problems as in 
the $\mathrm{BaVS}_{3},{ }^{35} \mathrm{Fe}_{3} \mathrm{O}_{4},{ }^{9}$ and $\mathrm{NaV}_{2} \mathrm{O}_{5} \cdot{ }^{26}$ In this latter case the charge disproportion was found to be very small $( \pm 0.045$ electron).

\section{Comparison between experiment and simulation}

The comparison between measured and simulated spectra is not yet a standard procedure in RXS and to the best of our knowledge the simultaneous fit of geometrical and electronic parameters has never been attempted before. Up to now, most studies in this field use simulations to check the origin of specific reflections or of peculiar features in one or several diffracted peaks.

The large number of parameters to refine makes the use of objective confidence factors to compare experiment and theory necessary. The spectra analyzed in RXS and in lowenergy electron diffraction (LEED) share some similitudes: same energy range for the (photo) electron, analogous number of reflection, and finally the same level of certitude. Thus it is natural to use the confidence factors already used in LEED in the analysis of our RXS data.

The first criterion is called $D_{1}$ (Ref. 36) and is given by

$$
D_{1}\left(I_{h k l}^{e}, I_{h k l}^{t}\right)=50 \int_{E_{\min }}^{E_{\max }}\left|I_{h k l}^{e}(E)-I_{h k l}^{t}(E)\right| d E,
$$

where $I_{h k l}^{t(e)}$ stand for the normalized reflection theoretical (experimental) intensity such that

$$
\int_{E_{\min }}^{E_{\max }} I_{h k l}^{e}(E) d E=1
$$

$E$ stands for the energy and $\left[E_{\min }, E_{\max }\right]$ is the energy range. For a set of reflections, the total $D_{1}$ is just the average of the individual $D_{1}$. Note that $D_{1}$ is a metric distance and is given in percent. A value of $D_{1}=12 \%$ means a pretty good agreement when the set is large. Smaller value (down to $5 \%$ ) can be obtained when all the spectra are of the same family.

Note that within the $D_{1}$ metric the information on the relative intensity between the different reflections is lost due to renormalization. Other criteria, which keep a unique normalization coefficient between experiment and theory, proved to be unsuccessful as it is explained in the next chapter. A second confidence factor, a $R$ factor called $R x$ by Horsky et al.,${ }^{37}$ was used as well. Its main difference with $D_{1}$ is that the latter is related to the square of the difference between the spectra. When both convergence criteria concur to the same model, the degree of confidence results is enhanced.

In classical $\mathrm{x}$-ray diffraction on monocrystal one compares peak intensities; in the powder case one compares rather simple peak profiles. Here we compare sets of simulated spectra with sets of experimental curves. It is thus very difficult to give an objective value for the standard deviation. Because of the numerical aspect, our technique is closer to LEED and we also use the technique employed in this field to give a value for the uncertainty. We simply define it for each parameter as the necessary value of its deviation to get an increase in the metric distance of $2 \%$.

\section{Simulations}

Simulations and fit of the experimental data have been carried out by using the FDMNES code. FDMNES (Ref. 38) is a monoelectronic code using either the full multiple-scattering theory (MST) or, alternatively, the finite difference method (FDM). Calculations can be classical, magnetic, or fully relativistic including the spin orbit. Different options for the exchange-correlation potential are possible, but most often the Hedin and Lundqvist ${ }^{39}$ energy-dependent approach is preferred. The code does not use the three-dimensional periodicity to calculate the final states. It solves the Dyson equation in a cluster centered around the different absorbing atoms. Then it calculates the energy dependence of the anomalous scattering factors of the corresponding elements. From these, following Eq. (4), the structure factor is calculated using eventually the space group symmetry operations. The intensity of the different peaks is then calculated. In the same code, the simulations can be performed by varying different crystallographic or electronic parameters along a multidimensional grid of parameters. They are compared with experimental spectra using the criteria $D_{1}$ or $R x$; the minimum value corresponds to the better set of parameters.

FDMNES is thus specially adapted to quantify the charge ordering as revealed in RXS experiments. For this problem the so-called non-muffin-tin effect is not very important, and the MST which is faster than the FDM has been chosen. Indeed simulations are rather time consuming [typically a MST calculation to get the atomic scattering factors (ASF) lasts a day on a cluster of ten processors], because they have to be performed in all the nonequivalent absorbing atoms. For each of them relatively big clusters (up to a radius of $7 \AA$, containing around 150 atoms) were used. To compare experiment and simulation we use a simple multidimensional grid of parameter. Fortunately the time-consuming part of the job, which is the evaluation of the ASF, is performed only one time. As it has been checked, the effect of the atomic displacements and charge disproportion is nearly contained in the Bragg factor and in the energy shift of the ASF. For each model, the calculation of the structure factors, of the Bragg reflection spectra, and finally of the confidence factors is very fast (around $1 \mathrm{~s}$ ). This makes a complete fit of the different parameters possible.

\section{DESCRIPTION OF THE STRUCTURE}

At room temperature magnetite displays a cubic structure of space group $F d \overline{3} m$ and lattice parameters $a_{c}=8.394 \AA$. It has two nonequivalent iron sites, one in a slightly distorted octahedral environment (Wyckoff site $16 d$, point group $\overline{3} m$ ) and the other in a tetrahedral coordination (Wyckoff site $8 a$, point group $\overline{4} 3 m$ ). In a pure ionic description the octahedral iron is in a +2.5 charge state; the tetrahedral one is +3 . The Verwey transition leads to a lowering of the symmetry of the unit cell, at least down to $C c$ but there are hints that it is probably triclinic. ${ }^{40}$ Despite numerous efforts and attempts in the past to refine the low-temperature structure only an approximate structure in a smaller unit cell has been completely refined. Until now the Pmca space group is supposed 
TABLE I. Pmca iron atom positions given in the $C c$ cell. Sites 1-16 first are octahedral positions, where sites 17-24 are tetrahedral sites. The prime for the Pmca index is to show the formally inequivalent sites due to the nonright $\beta$ angle. Their positions were nevertheless refined together as a unique parameter.

\begin{tabular}{|c|c|c|c|c|}
\hline$C c$ index & Pmca index & $x$ & $y$ & $z$ \\
\hline 1 & 1 & 0 & 0 & 0 \\
\hline 2 & 1 & 0 & 0.5 & 0 \\
\hline 3 & $1^{\prime}$ & 0.25 & 0 & 0 \\
\hline 4 & $1^{\prime}$ & 0.75 & 0 & 0 \\
\hline 5 & 2 & 0 & 0.7549 & 0.25 \\
\hline 6 & 2 & 0 & 0.2548 & 0.25 \\
\hline 7 & $2^{\prime}$ & 0.25 & 0.7452 & 0.75 \\
\hline 8 & $2^{\prime}$ & 0.25 & 0.2452 & 0.75 \\
\hline 9 & 3 & 0.125 & 0.88295 & 0.3801 \\
\hline 10 & 3 & 0.375 & 0.11705 & 0.6199 \\
\hline 11 & 3 & 0.625 & 0.126 & 0.3801 \\
\hline 12 & 3 & 0.875 & 0.874 & 0.6199 \\
\hline 13 & 4 & 0.125 & 0.126 & 0.37659 \\
\hline 14 & 4 & 0.375 & 0.874 & 0.62341 \\
\hline 15 & 4 & 0.625 & 0.2517 & 0.37659 \\
\hline 16 & 4 & 0.875 & 0.2483 & 0.62341 \\
\hline 17 & 5 & 0.125 & 0.2517 & 0.06366 \\
\hline 18 & 5 & 0.375 & 0.2483 & 0.93634 \\
\hline 19 & 5 & 0.625 & 0.2517 & 0.06366 \\
\hline 20 & 5 & 0.875 & 0.2483 & 0.93634 \\
\hline 21 & 6 & 0.125 & 0.00305 & 0.18867 \\
\hline 22 & 6 & 0.375 & 0.99695 & 0.81133 \\
\hline 23 & 6 & 0.625 & 0.00305 & 0.18867 \\
\hline 24 & 6 & 0.875 & 0.99695 & 0.81133 \\
\hline
\end{tabular}

to give the best agreement ${ }^{20}$ with experiment. The new unit cell is rotated by $45^{\circ},\left(F d \overline{3} m \rightarrow P m c a\right.$, and $a_{c} \times a_{c} \times a_{c}$ $\rightarrow \approx a_{c} / \sqrt{2} \times a_{c} / \sqrt{2} \times 2 a_{c}$ ) which generates six nonequivalent iron sites, four of them possessing a distorted octahedral environment. Each site possesses a multiplicity equal to 4 . Note that Wright et al..$^{20}$ found $\beta=90.2363^{\circ}$ that makes monoclinic $P 2 / c$ a better description of the unit cell. Under this symmetry lowering the two first octahedral iron sites split into four nonequivalent positions of multiplicity 2 .

In the present study we have measured peaks not allowed in the Pmca (or $P 2 / c$ ) and are compatible with the four times larger unit cell corresponding to the $C c$ space group. From the Pmca unit cell, the $C c$ unit cell has its $a$ and $b$ cell parameters doubled. Consequently the new unit cell parameters are $a=11.888814 \AA, b=11.849402 \AA, c$ $=16.775150 \AA, \alpha=90^{\circ}, \beta=90.2363^{\circ}$, and $\gamma=90^{\circ}$ with 24 nonequivalent iron atoms of multiplicity 4 (see Table I).

The relation between the cubic and the $C c$ and Pmca reflection index is given by

$$
\begin{gathered}
h_{C c}=2 h_{P m c a}=h+k, \\
k_{C c}=2 k_{P m c a}=-h+k,
\end{gathered}
$$

$$
l_{C c}=l_{\text {Pmca }}=2 l,
$$

where $h_{C c}, k_{C c}$ and $l_{C c}$ are the $C c$ indices, $h_{P m c a}, k_{P m c a}$ and $l_{P m c a}$ are the Pmca indices, and $h, k$ and $l$ the cubic ones. In agreement with most published studies the cubic indices notation will be kept throughout this paper. Reflections such that $h+k=$ odd do not exist in the Pmca unit cell and are thus pure $C c$ reflections. We shall call them in the following as the " $C c$ reflections."

\section{EXPERIMENT}

RXS experiments were carried out at the U.K. CRG beamline (XMaS) in the European Synchrotron Radiation Facility (Grenoble) operating at photon energies between 7.060 and $7.220 \mathrm{eV}$. The x-ray beam wavelength was selected by a $\mathrm{Si}(111)$ double crystal monochromator with an energy resolution of $0.8 \mathrm{eV}$ at the photon energies of the experiment. After scattering an analyzer crystal was used to filter out background coming from the sample (mostly fluorescence). The two samples used have been prepared using the vertical floating zone technique, with the help of an optical furnace (Crystal System Inc. FZ-T-10000-H-VI-P-G). The $\mathrm{Fe}_{3} \mathrm{O}_{4}$ feed rods were prepared from high purity powder (Alfa Aesar 99.997\%), compacted into cylindrical shape under isostatic press (up to 1200 bars). The Verwey transition was characterized by magnetic susceptibility measurements using SQUID and results have been given by Delille et al. ${ }^{41}$ The as-grown crystals exhibit a sharp bulk Verwey transition with $T_{V}=121 \mathrm{~K}$. The scattering experiment was carried out in reflexion mode, off a $\{110\}$ surface for both samples and at $50 \mathrm{~K}$.

RXS experiments must be performed on a single crystal, if a good signal-to-noise ratio is required. Traditionally one of the major difficulties of crystallographic work on magnetite has been to overcome a very strong self-absorption, twinning, extinction, and the presence of multiple scattering. These issues can be mastered in RXS studies more easily than in conventional single-crystal diffraction experiments. Indeed, parameter refinement in the former is carried through the analysis of the energy dependence of the photons scattered within each of the measured Bragg reflections, the absolute scale being an unrefined proportionality constant. In order to minimize the number of crystallographic domains, a magnetic field of $0.3 \mathrm{~T}$ has been applied parallel to one of the [001] directions that uniquely defines the $c$ direction. In the previous work we found that all reflections can be indexed within a single domain. Multiple-scattering events are eliminated by the measurement of the energy spectra at different azimuths and crystal settings. The absorption correction is carefully performed by using the energy dependence of the intensities of several very strong Bragg reflections as a measure of absorption. Three different strong reflections $\left[I_{\text {ref }}(\vec{Q}, \omega)\right],(4,0,0),(4,4,4)$ and $(2,2,0)$, have been used to extract a good measure of the absorption coefficient. The anomalous contribution of these reflections can easily be computed and reinjected back into the corresponding structure factor to finally deduce the absorption: absorption $(\omega)$ $\approx I_{\text {ref }}(\vec{Q}, \omega) /|F(\vec{Q}, \omega)|^{2}$. The data presented in this paper already include the absorption correction. 


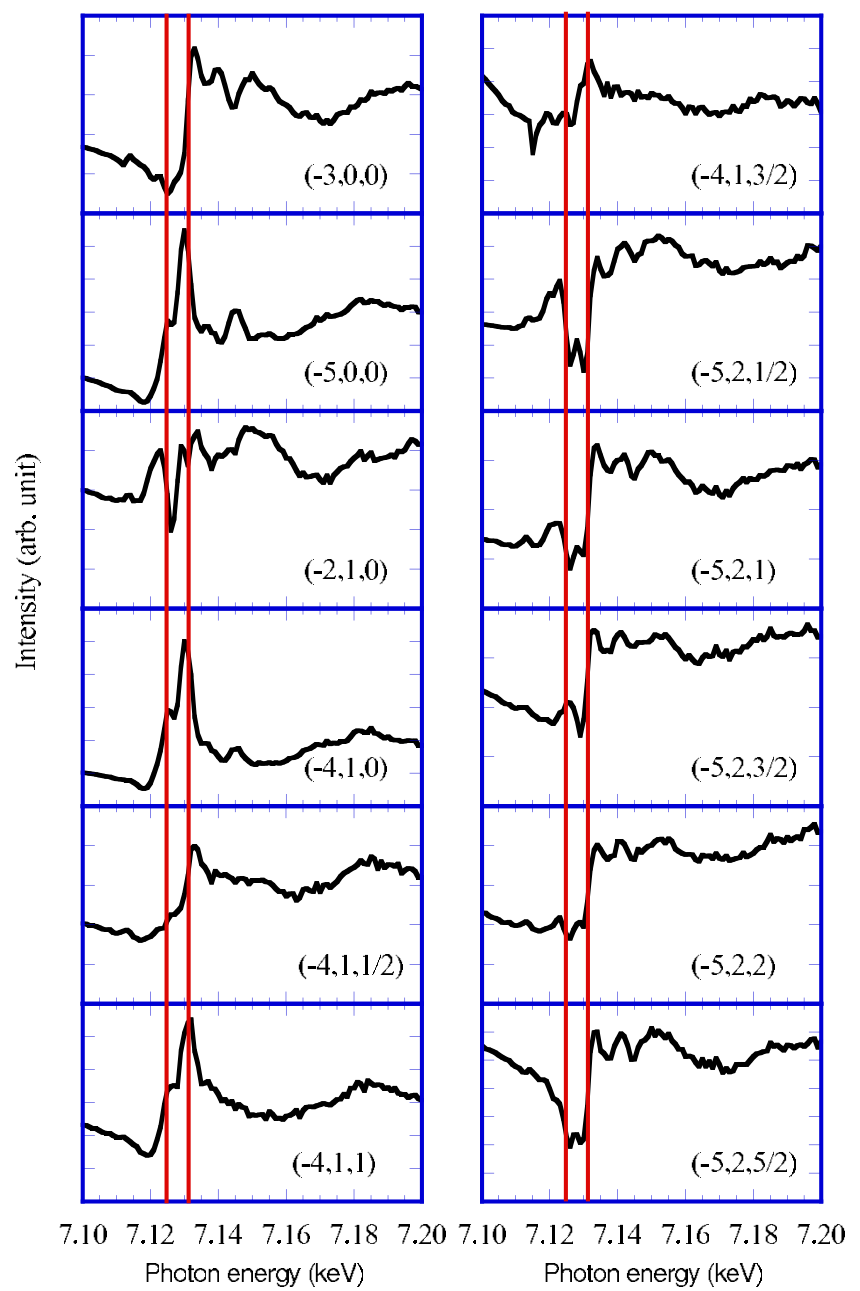

FIG. 2. (Color online) Energy variation of the intensities at the peak for a number of Bragg reflections observed only within the $C c$ space group $(h+k=$ odd $)$ around the Fe $\mathrm{K}$ edge and at $T=50 \mathrm{~K}$. In this figure and in the following, the reflections are indexed according to the Miller indices of the high-temperature cubic phase. The reflections are corrected from self-absorption. The vertical red lines show the position of the features supposed to be a signature of the charge ordering phenomena.

\section{DESCRIPTION OF THE DATA}

In Fig. 2 we show the measured reflections with $h+k$ $=$ odd. These reflections have a null structure factor in the Pmca space group and are indeed indexed in the $C c$ space group. For comparison some of the reflections indexed within the Pmca space group $(h+k=$ even) are shown in Fig. 3 . Both sets of reflections can be grouped into different families depending on their global shape. A very important point is that all $h+k=$ odd reflections display the typical two-peak feature which has been acknowledged in the previous study ${ }^{9}$ as a signature of the charge ordering. These two features at 7.125 and $7.131 \mathrm{keV}$ sometimes appear as peaks and sometimes as hollows reflecting the fact that the coefficient affecting the derivative of the scattering factor [Eq. (6)] can change sign as a function of $\vec{Q}$ and consequently the charge ordering effect sometimes adds to the geometrical structure effect and sometimes subtracts to it. We can infer from this

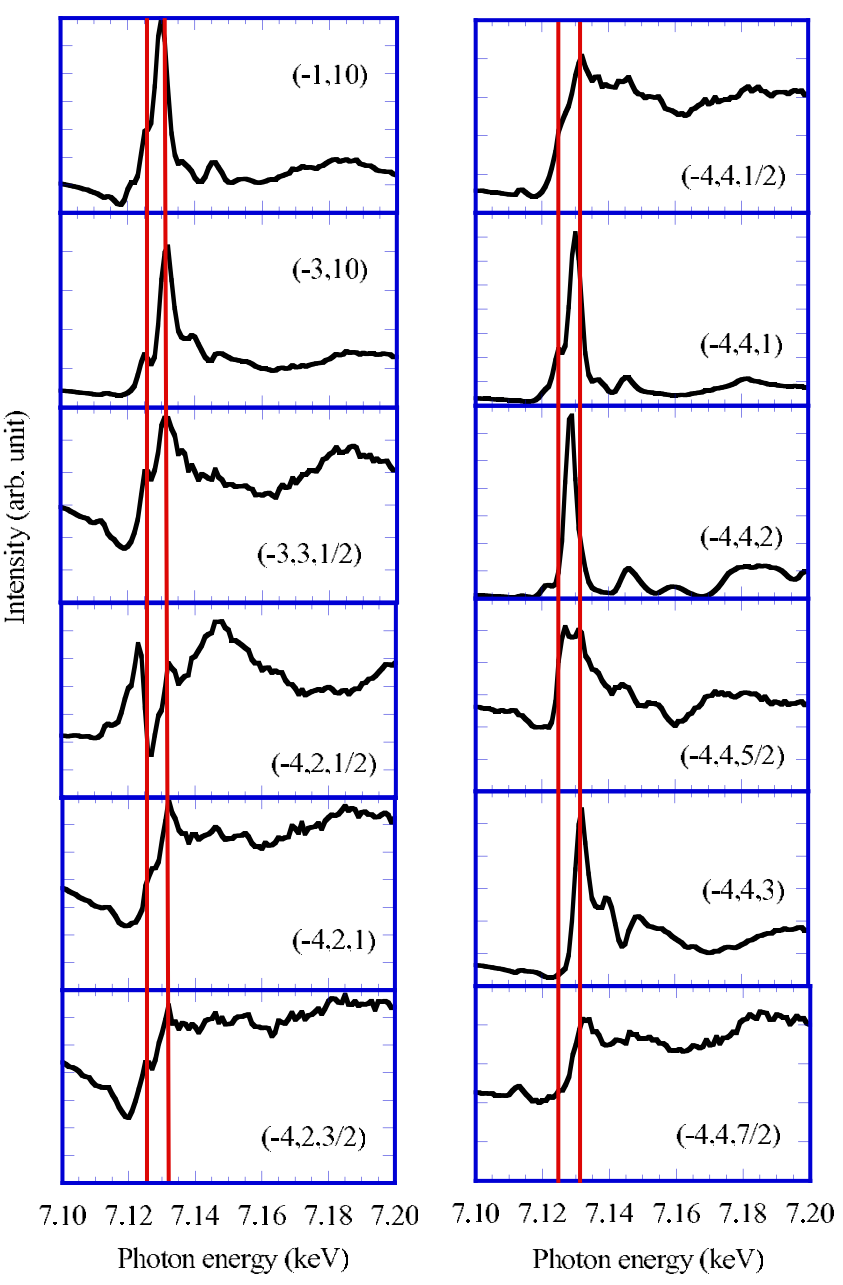

FIG. 3. (Color online) Energy variation of the intensities at the peak for a number of Bragg reflections observed within the Pmca space group $(h+k=$ even) around the $\mathrm{Fe} \mathrm{K}$ edge and at $T=50 \mathrm{~K}$. The reflections are corrected from the self-absorption. The red vertical lines show the position of the features supposed to be a signature of the charge ordering phenomena. The main peaks for some reflections is directly related to the charge ordering process. For some other reflections like the $(-4,4,2)$, the main peak at 7.128 $\mathrm{keV}$ is due to the trigonal field at the octahedral sites and thus their anisotropy has nothing to do with charge ordering.

result that the different supposedly equivalent iron atoms in the Pmca space group have in fact some charge disproportions among them.

Some of the reflections were measured at different azimuths. They have not revealed very strong angular dependencies of the shape of the spectra. On the contrary the average intensity for some reflections displays an abrupt jump at some angle, and this without changes in the spectrum shape. This fact has no relation with the known azimuthal dependence of the Templeton ${ }^{30}$ scattering and we concluded it is the signature of the presence of microcrystallites. We cannot exclude that on turning the sample around (or on changing the reflection) the photon beam jumps from one grain to another with the immediate consequence that the information on the absolute intensity becomes unreliable. We have not pursued further the study of the microstructure as it 
is another unsettled problem in $\mathrm{Fe}_{3} \mathrm{O}_{4}$ that we merely acknowledge. Thus the analysis with the metric distance is performed with individual coefficient for each reflection instead of using a global one for all reflections. The general trend for the intensities for the different families of reflections remains valid: $C c$ reflections are less intense than the Pmca ones and these are weaker than the cubic ones.

\section{STRATEGY AND SENSITIVITY}

\section{A. First approximations}

It is evident from the considerations above that the number of parameters to fit is very large. Including the 32 oxygen atoms, one gets $56 \times 3$ position parameters, plus the charge of all these atoms. In the following we discuss the different approximations that we have to make in order to pursue the analysis of our data.

\section{Oxygen contribution}

The RXS experiments performed around the iron edge are especially sensitive to these sites. However, the oxygen atom positions and displacements contribute to the scattering cross section in two different ways. First, as shown in Eq. (6) they participate to the scattering cross section with a constant factor, different for each reflection. Second, they change slightly the anomalous scattering factors, as seen in the Pmca distortion of the cubic cell. Nevertheless $C c$ reflections are weaker than those indexed in the Pmca unit cell and therefore the concomitant atomic displacements ought to be smaller. We can safely conclude that the relative displacement of the oxygen atoms will not affect much the total scattering factor. This point has been verified to hold true and it is further discussed below. In a first approximation we have thus assumed this contribution to be very small and we have disregarded it from the calculations. Only the known oxygen positions in the Pmca unit cell have been kept in the simulations.

\section{Tetrahedral iron}

Charge ordering takes place at the octahedral sites. Moreover the tetrahedral environment endorses less possibility for distortion and we can suppose a priori that these sites have very little influence on the intensity of the $C c$ reflections. The measured spectra (Figs. 2 and 3) show that the charge effect is present with features at the same energies in the $C c$ reflections and in the Pmca one. This contrasts with the position of the absorption edges of both sites (Fig. 1) which disregards the possibility for a charge effect occurring at the same energy as in the octahedrally coordinated iron. We can conclude that charge ordering at the tetrahedral site is negligible. RXS experiments at the Fe $L$ edges have come up with the same conclusion. ${ }^{13}$ Despite these qualitative remarks, we have made some tests which confirm these considerations as is shown in Sec. VIII.

\section{Octahedral iron}

In our previous paper on the charge disproportion ${ }^{9}$ we obtained different results on the two pairs of octahedral iron sites. The charge disproportion between the $\mathrm{Fe}_{1}$ and $\mathrm{Fe}_{2}$ sites was estimated with small error bar $\mp(0.12 \pm 0.025)$ around +2.5 . A stronger uncertainty was found in the charge disproportion between the $\mathrm{Fe}_{3}$ and $\mathrm{Fe}_{4}$ sites. For this second pair the $C c$ unit cell was used in the refinement yielding a charge disproportion of $\pm(0.10 \pm 0.06)$ around +2.5 . We explained this larger uncertainty as due to the different environment of the two pairs of octahedral irons. Whereas $\mathrm{Fe}_{1}$ and $\mathrm{Fe}_{2}$ are at the centers of their respective octahedron, $\mathrm{Fe}_{3}$ and $\mathrm{Fe}_{4}$ possess a more distorted local environment; the iron atoms are slightly off center. Furthermore these position shifts are responsible for the doubling of the cell along the $c$ axis whereas the $\mathrm{Fe}_{1}$ and $\mathrm{Fe}_{2}$ ordering gives no effect on the cell doubling.

An improvement on the structure and charge determination is expected from the analysis of the position of these off-centered Fe sites and we shall focus our study on the analysis of these sites. This simplification along with that on the tetrahedral and on the oxygen sites makes the number of parameters decrease down to 32 , which includes the charge and the position along the three directions of each of the eight $\mathrm{Fe}$ atoms at sites $\mathrm{Fe}_{3}$ and $\mathrm{Fe}_{4}$. If we additionally assume that Pmca is a good overall structure of the displacement pattern the number of free parameters can be further reduced. Implementing this assumption comes to consider that the sum of the atomic displacements of the atoms in positions 9-10-11-12 (that makes the $\mathrm{Fe}_{3}$ site in Pmca) must add to zero, and the same should hold for the subset 13-14-15-16 (that makes the $\mathrm{Fe}_{4}$ site in Pmca). Moreover the average disproportion among the eight atoms is kept to zero in order to maintain a global charge neutrality. At the end one remains with 23 independent parameters.

\section{B. Sensitivity}

Slight changes in position or charge of the $\mathrm{Fe}_{n}$ sites, with $n$ running from 9 to 16 , yield an important contribution to reflections indexed in the $C c$ cell, as expected. Conversely, the $h+k=$ even reflections display hardly any effect except at some very weak ones such as the $\left(-4,2, \frac{1}{2}\right)$. The fit and the ensuing discussion will be performed only with the spectra at these $C c$ reflections.

As a first test we distort the Pmca symmetry to reach the $C c$ by a simple displacement in opposite direction of two previously equivalent atoms $\left(\mathrm{Fe}_{9}\right.$ and $\left.\mathrm{Fe}_{11}\right)$ along $a$. The result is that all the $C c$ reflections have very similar shapes; the difference is merely a multiplicative factor (see Fig. 4). We have checked that the same kind of spectra is obtained for any other pair of atoms. The agreement is not good when comparing with the experiment except for some reflections where the global shape could appear satisfactory.

A second test consists in setting a charge ordering on the same pair of atoms without any displacement. For this purpose a simple shift of $\pm 0.2 \mathrm{eV}$ of the structure factor is applied. From our calculations this corresponds to a \pm 0.025 charge disproportion (or $\mp 0.025$ in number of electrons). The two-peak feature already mentioned at 7.125 and 7.131 $\mathrm{keV}$ (Fig. 4) is obtained but with almost no intensity outside this energy range. The overall shape is very different from 

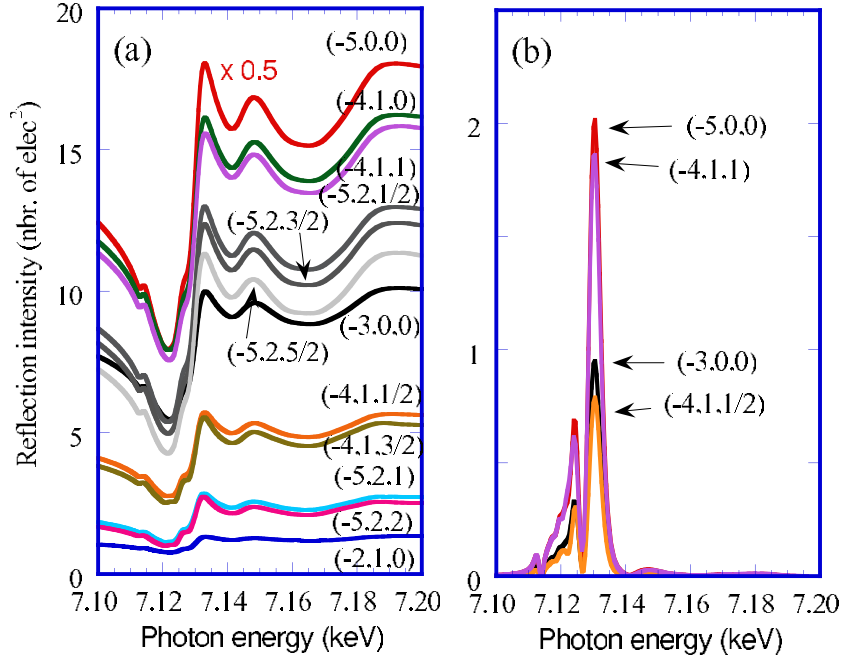

FIG. 4. (Color online) Energy variation of the simulated intensities of the Bragg reflections corresponding to the $C c$ space group around the Fe K edge. The spectra on the left side (a) are obtained with a displacement of \pm 0.002 cell unit along $a$ for the $\mathrm{Fe}_{9}$ and $\mathrm{Fe}_{11}$ sites and no charge disproportion. They all have almost the same shape, the difference is mainly a multiplicative factor, no one shows the double feature at 7.125 and $7.131 \mathrm{keV}$. The spectra on the right side (b) are obtained with a charge disproportion of \pm 0.025 electron for the $\mathrm{Fe}_{9}$ and $\mathrm{Fe}_{11}$ sites but without any displacement from the Pmca positions. They all show almost only the double feature at 7.125 and $7.131 \mathrm{keV}$. Note that the relative amplitude between spectra is different with the relative amplitude of the spectra obtained with the sole displacement as shown in (a). In this figure and in the following the intensity unit is the square of number of electron. Relative amplitudes can thus be compared.

that of the measurements showing that charge disproportion ought to be accompanied with a Fe displacement. This conclusion stems from the form of Eq. (6).

A third test consists in jointly performing the displacement along $a$ and an energy shift. As it is shown in Fig. 5 the agreement is good for some reflections but bad for some others. Both effects seem to add for some reflections and to subtract for some others. Reversing the sign of the shift without inverting the sign of the atomic displacement produces a swap in the quality of the simulation. We have repeated this simulation on other pair of atoms and the result is always the same. The conclusion is that it is not possible to improve all the spectra with a single pair of atoms irrespectively of the atomic displacement direction, that is along $a, b$ or $c$. The intensity of the spectra is roughly proportional to the square of the displacement in the energy range where this effect is dominant and proportional to the square of the charge disproportionation in the two-peak region as it was expected from formula (6). By playing these tricks we reach a first conclusion on the relative amplitude of the displacement and on the magnitude of the charge disproportionation required to obtain spectra in close agreement with the experiment. Roughly, a 0.025 electron charge disproportion must correspond to a $0.02 \AA$ displacement. When fitting the charges and displacements for all the atoms, their values can vary in different ways. Nevertheless the average of the relative value of both parameters must remain of this order of magnitude.

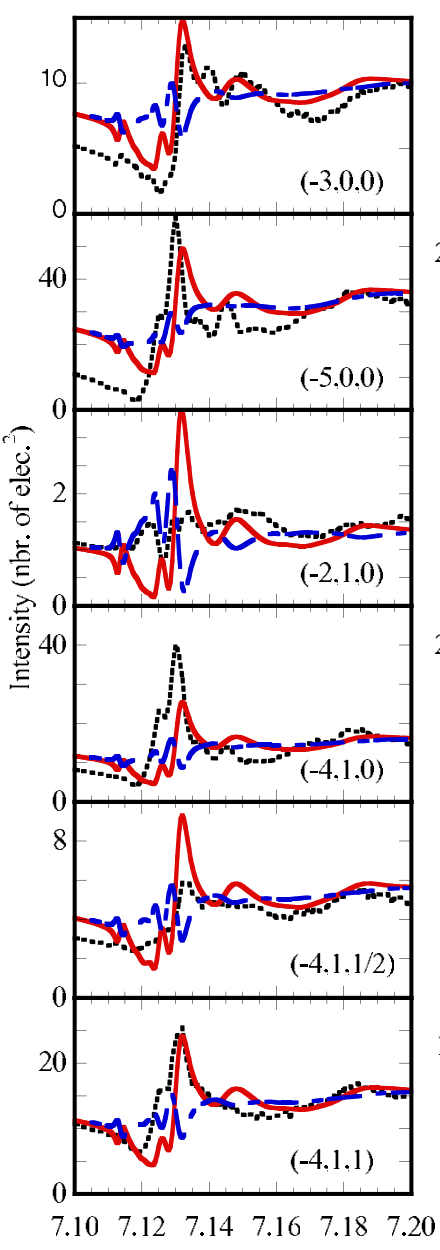

Fhotcr energy (keV)

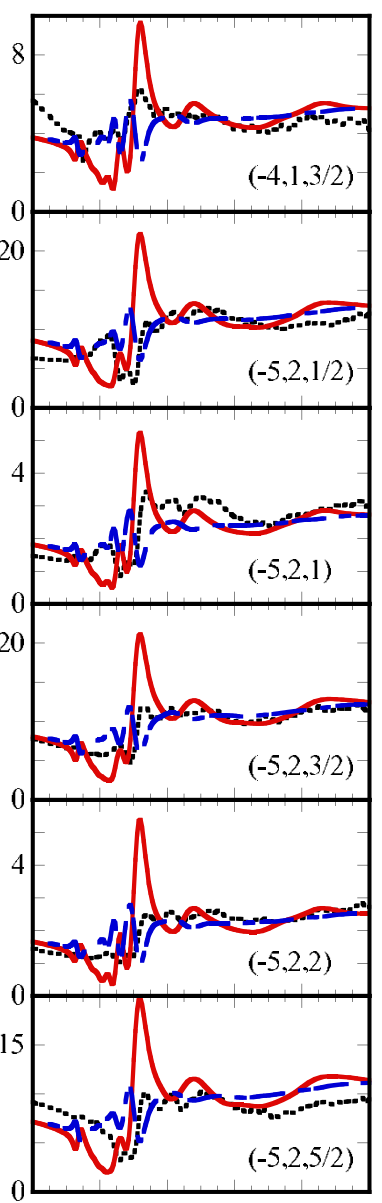

$\begin{array}{llllll}7.10 & 7.12 & 7.14 & 7.16 & 7.18 & 7.20\end{array}$ Frctcr enercy (keV)
FIG. 5. (Color online) Energy variation of the simulated intensities and the corresponding experiment (dotted) of Bragg reflections existing in the $C c$ space group. These spectra are obtained with two opposite charge disproportions of respectively \pm 0.025 (full line) and $\mp 0.025$ (dashed line) and a same displacement of \pm 0.002 cell unit along $a$ for the $\mathrm{Fe}_{9}$ and $\mathrm{Fe}_{11}$ sites. Some reflections have a good shape but some others are worth that without charge disproportion. A multiplicative factor is applied on the experimental spectra to compare with the simulations.

It has been very difficult to find a pattern of charge and displacements which permits to improve all the measured spectra. We have also tried to use a simplified pattern for the charges and for the displacement direction, but we have not succeeded in this way. To get a reasonably good agreement with measurements we need to apply different displacements on the different pairs of iron atoms with different shifts.

A constraint that can be used to reduce further the number of possible models is the occurrence of a net electric moment along the $a$ direction. ${ }^{21}$ For this to occur charge disproportion on the different pairs of iron sites (9-11), (10-12), (13-15), and (14-16) has to be of opposite sign. Thus we keep this rule for all the parameters, charges and displacements are opposite on all these pairs of sites. We are left with 16 independent parameters; the most relevant feature is the relative way of displacement among the atoms. At this level of the discussion we have decided to keep the modulus of the dis- 
placements constant for all the atoms, an approach that does not seem unnatural in view of the underlying cubic symmetry. The magnitude of the displacement can nevertheless be different along the three directions.

It is then possible to use a simple multidimensional grid of parameters, because there are only three values for each parameter that we can correlate, atom by atom, throughout the entire $C c$ cell. In addition we are sure to avoid the occurrence of a local minimum of the metric distance and that another set of parameters gives a satisfactory account of the spectra.

The $C c$ reflections are sensitive to the absolute value of the displacement mostly only through a constant factor in the intensity; the shape of the spectra is not modified. Within our procedure of using normalized intensities, the absolute value of the displacements is not a critical parameter. Its magnitude can be quantified in some very weak Pmca reflections as the $(-4,4,2)$. Indeed the Thomson part of the amplitude is of the same order of magnitude as the anomalous Templeton part, ${ }^{30}$ and both contributions are not modified in the same way when displacing the atoms from their positions in the Pmca unit cell. Consequently, small changes in position are easy to detect at these reflections, and they can eventually permit the estimation of its maximum value.

In the first runs only the pattern of displacement along the three directions and the charge disproportions are refined using only the $C c$ reflections. Then the magnitudes of atomic displacement and of the charge ordering of the $\mathrm{Fe}_{1}-\mathrm{Fe}_{2}$ Pmca sites have been fitted within this pattern.

\section{RESULTS}

Calculations and comparison with experiment have been performed on a wider energy range $(90 \mathrm{eV})$ than in the previous study. ${ }^{9}$ We got the very satisfactory result that the best agreement given by the metric distance corresponds to a very good visual accord of all the different spectra. For all of them charge and displacement effects add or subtract conveniently. The best result is given in Fig. 6 and the refined parameters (position and charge of the atoms) are given in Table II and Fig. 7. In these calculations atomic form factors are calculated in the Pmca structure; the $C c$ displacements are taken into account only through the Bragg factor. In a second step new structure factors are calculated by using the so-refined $C c$ unit cell, and the calculated spectra show no difference on using the scattering form factors of either the Pmca or the $C c$ structures. Again we are assuming that the displacements of the oxygen atoms hardly affect the diffracted intensities, at least for the $C c$ reflections, and do not introduce subtle changes in the atomic form factors.

The charge ordering on the $\mathrm{Fe}_{1}-\mathrm{Fe}_{2}$ sites remain at $\mp 0.10$, very close to our previous results. ${ }^{9}$ The $D_{1}$ metric distance and the $R x$ confidence factor have reached the same result. This gives more confidence to the model that we have described here.

Following Goff et al., ${ }^{10}$ we have considered a possible charge disproportion between the $\mathrm{Fe}_{1}$ and $\mathrm{Fe}_{1}$, sites and the $\mathrm{Fe}_{2}$ and $\mathrm{Fe}_{2}$, sites. We found a slightly better agreement when the charge disproportion is -0.125 and -0.075 for the

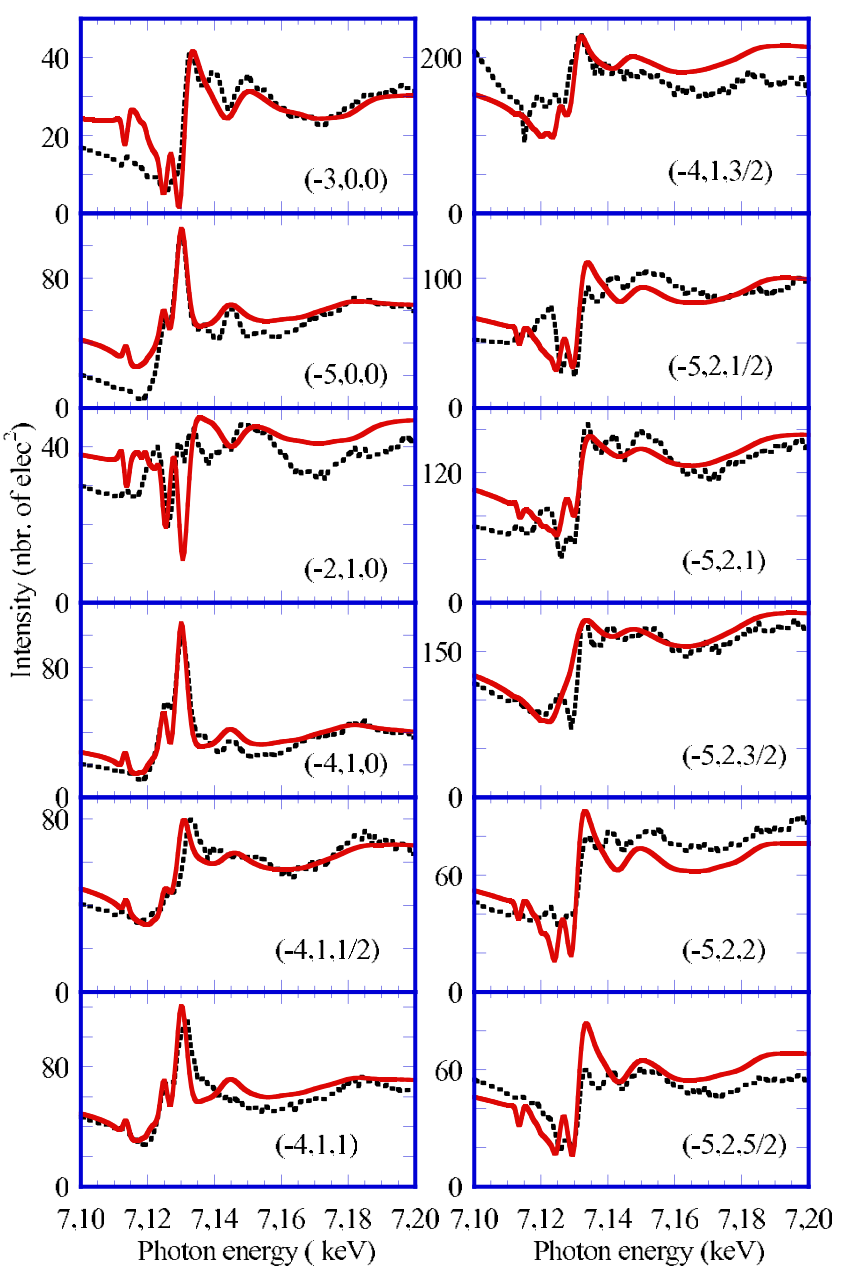

FIG. 6. (Color online) Energy variation of the simulated intensities (full line) and measured spectra at $50 \mathrm{~K}$ (dotted) of Bragg reflections corresponding to the $C c$ space group around the $\mathrm{Fe} \mathrm{K}$ edge. The simulation is performed with the structural and charge model given in Table II.

first pair and unchanged, +0.10 , for the second pair. This variation is of the same order of magnitude than the estimated uncertainty and we can conclude that the variation of charge disproportion, if any, ought to be very small between these sites.

TABLE II. Fitted iron atom positions and charges given in the $C c$ cell. They are defined in the same way as in Table I. The position, in cell units, is relative to the Pmca structure. The charge disproportion, $\delta$ charge, is relative to +2.5 .

\begin{tabular}{crrrr}
\hline \hline$C c$ index & \multicolumn{1}{c}{$\delta x$} & \multicolumn{1}{c}{$\delta y$} & $\delta z$ & $\delta$ charge \\
\hline 9 & 0.0020 & 0.0015 & 0.000 & 0.025 \\
10 & 0.0020 & 0.0015 & 0.000 & 0.050 \\
11 & -0.0020 & -0.0015 & 0.000 & -0.025 \\
12 & -0.0020 & -0.0015 & 0.000 & -0.050 \\
13 & 0.0020 & 0.0015 & 0.000 & 0.000 \\
14 & -0.0020 & -0.0015 & 0.000 & 0.025 \\
15 & -0.0020 & -0.0015 & 0.000 & 0.000 \\
16 & 0.0020 & 0.0015 & 0.000 & -0.025 \\
\hline \hline
\end{tabular}



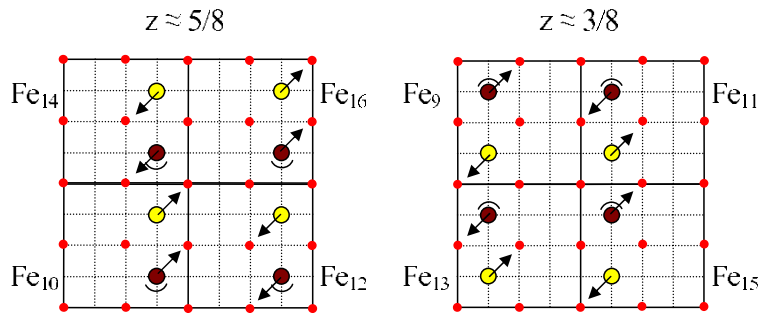

FIG. 7. (Color online) Pattern of displacements of the iron atoms corresponding to Table II. Only the atoms close to the planes $z=3 / 8$ and $z=5 / 8$ are shown. Each site corresponds to four equivalent positions, two in each plane obtained by $(x, y, z) \rightarrow(x+1 / 2, y$ $+1 / 2, z)$; the two other atoms are at $z \approx 1 / 8$ and $z \approx 7 / 8$ and the displacement pattern can be obtained by the symmetry operations $(x, y, z) \rightarrow(x,-y, z+1 / 2)$ and $(x+1 / 2,-y+1 / 2, z+1 / 2)$. The iron indexes correspond to the one used in the $C c$ unit cell. In brown and yellow are shown respectively the $\mathrm{Fe}_{3}$ and $\mathrm{Fe}_{4}$ as noted in Pmca; in red are the oxygen atoms. The arcs of circle give the direction of the main displacements from the cubic positions toward the Pmca ones. The arrows give the $C c$ displacement directions of the atoms from the Pmca positions. They are mainly toward one of the neighboring oxygen atoms in the plane. The four times smaller Pmca unit cell is also shown.

\section{DISCUSSION}

In the previous paper we have found that the charge ordering is very robust on the $\mathrm{Fe}_{1}-\mathrm{Fe}_{2}$ sites and we confirm this result. ${ }^{9}$ The new result \pm 0.10 electrons, to compare with \pm 0.12 electrons in our previous work, is within the uncertainty ( \pm 0.025 electron). Not surprisingly, we have found a more complex charge ordering pattern on the $\mathrm{Fe}_{3}-\mathrm{Fe}_{4}$ sites (as noted in Pmca) once the $C c$ superstructure model is considered. The average charge disproportion on these sites is smaller than in the previous study, around \pm 0.025 electron to compare with \pm 0.10 electron but a rather large uncertainty ( \pm 0.06 electron). The value of the charge disproportion seems small but the effect can nevertheless be quantified. Figure 8 shows some of the $C c$ reflections with and without the charge effect. It can be seen that the effect is important not only at the two double structure energy range but also at higher energies. This is due to the fact that these reflections are very weak.

The uncertainty in the Fe atomic displacement in the $a-b$ plane is around \pm 0.001 in cell units, and probably larger along the $c$ direction. We found the pattern of displacements very robust when both charge and displacement are considered simultaneously. We think that such a pattern will be a useful starting point for future studies of magnetite. The small amplitude of the obtained displacements from the Pmca structure makes also the results not in contradiction with the Wright et al. ${ }^{20}$ analysis. We show some of the Pmca reflections with and without the $C c$ displacement. This is shown in Fig. 9.

To illustrate the sensitivity of the very weak $(-4,4,2)$ reflection on the amplitude of the displacements we show in Fig. 10(a) the effect of no displacement, (b) the best model, and (c) a model with too large displacements (three times the best for all the sites, which is up to 0.006 unit cell). We recall

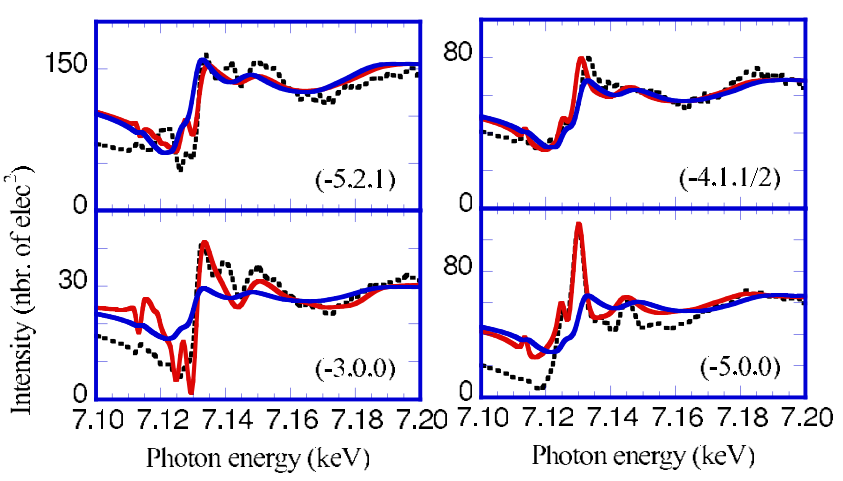

FIG. 8. (Color online) Energy variation of the intensities at the peak for Bragg reflections corresponding to the $C c$ space group around the $\mathrm{Fe} \mathrm{K}$ edge and at $T=50 \mathrm{~K}$. Dotted lines are the experiment and full lines are the simulations using the $C c$ displacements. The effect of the charge is clearly seen; the curves closer to the experiment include the charge disproportion, the other ones do not. For some reflections as the $(-3,0,0)$, the charge order effect is specially strong.

that such reflection already present in the high-temperature phase has a very different origin than the Pmca and $C c$ reflections. In this case, when pairs of equivalent atoms related by rotation symmetry have their Bragg factor opposite in sign, the anisotropy of the ASF makes the resulting structure factor not zero. This type of reflection is more difficult to fit, because all the complexities of the anisotropy and of the $C c$ symmetry breaking add up.

We come back to the a priori considerations concerning the possible influence of the other atoms on the diffracted $C c$ intensities. We already notice that the small displacements of the iron atoms in $C c$ do not change sufficiently their anomalous scattering factor to modify the calculated intensity. The same occurs for the surrounding oxygen atoms because the important parameter is the relative position of both species. The constant factor resulting from the Thomson part must be smaller than the one due to the iron atoms. It is difficult to
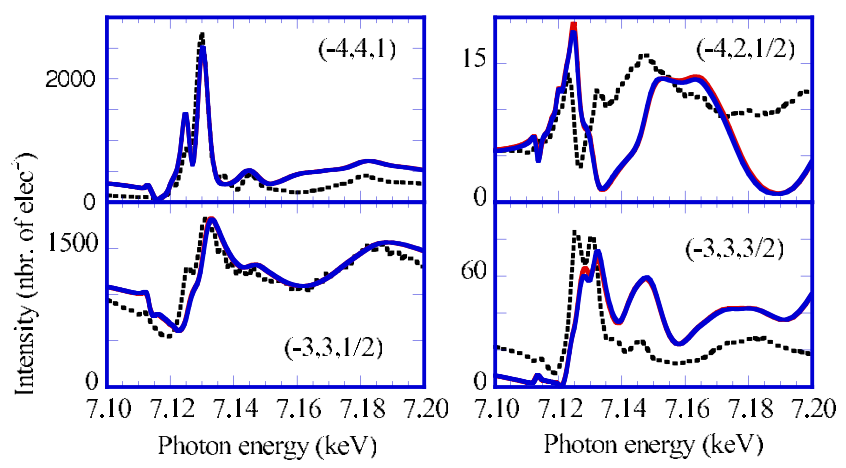

FIG. 9. (Color online) Energy variation of the intensities at the peak for a number of Bragg reflections corresponding to the Pmca space group around the $\mathrm{Fe} \mathrm{K}$ edge and at $T=50 \mathrm{~K}$. Dotted lines are the experiment and full line the simulations. There is almost no difference between the simulation including the $C c$ superstructure model and the pure Pmca model. Spectra corresponding to both models are very similar. The agreement remains less good for the very weak reflections. 


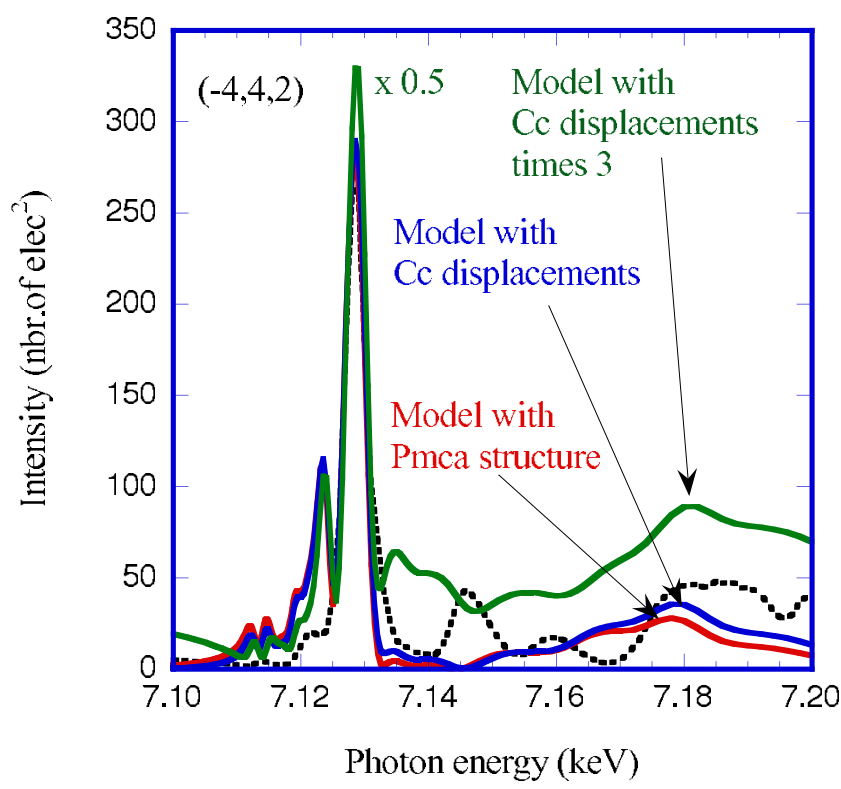

FIG. 10. (Color online) Energy variation of the simulated intensities (full line) and corresponding experiment (dotted) of the $(-4,4,2)$ Bragg reflection present in the Pmca (and also in the high-temperature cubic phase) space group around the Fe K edge. The three curves are the result of models (a) with no displacement, (b) the best displacement model (very close to the previous one) and (c) with a larger displacement model.

imagine that adding a relatively small constant can change drastically the shape of the $C c$ spectra and thus the conclusion on the pattern and charge. In the same way as for the octahedral sites, we have carried out a simulation of the charges and of the displacements at the tetrahedral Fe sites. In this case, keeping the octahedral sites at their original Pmca positions, we always found a poor agreement. Charge ordering at these sites can be ruled out. Some displacements remain possible but the agreement is poorer than on considering the octahedral site. We show in Fig. 11 the best agreement reached for two peaks; the spectra have mostly the same shape. Moreover it is not possible to get the peak at $7.125 \mathrm{keV}$. Some peaks as the $(-5,2,5 / 2)$ display a relatively good agreement, but the majority of them cannot reproduce the experiment. Thus small displacements on these
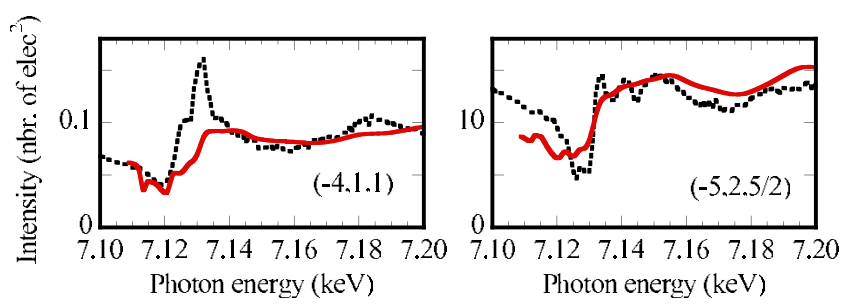

FIG. 11. (Color online) Energy variation of the simulated intensities (full line) and corresponding experiment (dotted) for two Bragg reflections of the $C c$ space group. The two simulated curves result from the fit of the displacements and of the charge on the tetrahedral sites. No charge disproportion is found. The displacement permits to get a satisfactory agreement only for some reflection as the $(-5,2,5 / 2)$, but it is never possible to get a good agreement on the majority of the reflections. sites cannot be completely excluded, although their contribution is not going to be sizable on the majority of the measured peaks. Therefore the conclusion for the $\mathrm{Fe}_{9}-\mathrm{Fe}_{16}$ octahedral sites remains valid.

The case of the $\mathrm{Fe}_{1}-\mathrm{Fe}_{8}$ octahedral iron sites because their atomic anomalous form factors are close to the $\mathrm{Fe}_{9}-\mathrm{Fe}_{16}$ ones and a fit on their parameters could eventually give an artificial good agreement. We did nevertheless simulations also at these sites to fit, their charges and displacements, keeping the other iron sites at their original Pmca positions. We found a surprisingly poor agreement, showing that charge disproportions and displacements at these sites must also be smaller than at the $\mathrm{Fe}_{9}-\mathrm{Fe}_{16}$, as it was expected. To illustrate this, the metric distances for the best models obtained by the three fits on the three groups of iron sites are as follows: $\mathrm{Fe}_{17}-\mathrm{Fe}_{24}$ and $\mathrm{Fe}_{1}-\mathrm{Fe}_{8}$ give, respectively, $6.16 \%$ and $5.87 \%$ to compare with $4.86 \%$ for the $\mathrm{Fe}_{9}-\mathrm{Fe}_{16}$ sites. Such a difference in the metric distance is a robust proof of our model. As for the tetrahedral iron sites, we cannot exclude that weaker charge disproportions and displacements exist on the $\mathrm{Fe}_{1}-\mathrm{Fe}_{8}$ sites.

From all these simulations, we do not pretend having solved completely the $C c$ structure. A complete quantitative evaluation of all these parameters would need a concomitant fit of all of them, a situation that is not possible today. The points we think safe in our model are the following: (1) the pattern of displacement of the $\mathrm{Fe}_{9}-\mathrm{Fe}_{16}$ sites with the corresponding charge disproportions, (2) the absence of charge disproportion on the tetrahedral sites, (3) the displacements of the $\mathrm{Fe}_{1}-\mathrm{Fe}_{8}$ and $\mathrm{Fe}_{17}-\mathrm{Fe}_{24}$ sites and the charge disproportions of the $\mathrm{Fe}_{1}-\mathrm{Fe}_{8}$ sites are smaller than the ones of the $\mathrm{Fe}_{9}-\mathrm{Fe}_{16}$ sites. Starting from our best model, a fit of the parameters of the other iron sites would lead to a model with uncertainties larger than their variation from the original Preca model.

An important unresolved issue is whether the charge ordering in oxides is driven by electrostatic repulsions between the charges (Wigner crystallization) or by the strains arising from electron-lattice interactions (such as Jahn-Teller distortions) involving different localized electronic states. For magnetite we obtained different values of charge disproportions for octahedral iron positions. These results agree with nuclear magnetic resonance ${ }^{7}$ and Mössbauer ${ }^{8}$ probes of the iron hyperfine fields that revealed many distinct components below $120 \mathrm{~K}$ that are associated with more than two charge configurations, intermediate between $\mathrm{Fe}^{+2}$ and $\mathrm{Fe}^{+3}$. Moreover the observed coupling effect of the $\mathrm{CO}$ and the geometrical distortions for positions of the types $\mathrm{Fe}_{3}$ and $\mathrm{Fe}_{4}$ is in agreement with the work of McQueeney et al. ${ }^{19}$ They have observed a large gap in the acoustic spin wave branch of magnetite below the Verwey transition. It was concluded that in the monoclinic phase, the spin wave would be affected by charge ordering and small crystalline distortions, because both these factors modify the superexchange.

Considering the charges and positions, we obtain a total electric polarization of $1.5 \mu \mathrm{C} / \mathrm{cm}^{2}$ along the $a$ direction. Previous studies have given the orientation of the moment, not its value. So we can just argue that the obtained value is reasonable as compared to known values in ferroelectric compounds. 


\section{CONCLUSION}

We have modeled the low-temperature structure of magnetite within a $C c$ unit cell and compared our simulations with resonant $\mathrm{x}$-ray scattering data, in an attempt to further pursue the complete resolution of the structure below the Verwey transition temperature. Starting from the structure proposed by Wright et al., ${ }^{20} \mathrm{Pmca}$, we have inferred a pattern of displacements that is in agreement with the $C c 2$ $\times 2$ larger unit cell. In addition, and by taking advantage of the possibilities of RXS concerning the determination of the charge, we have completed the charge ordering pattern previously initiated in Ref. 9. Our work shows also that quantitative RXS is a useful complementary tool helping in the resolution of structures when classical X-ray and neutron diffraction are not able to reach alone the final solution. We have shown here that using all the spectra shape around the edge, we can give new pieces of information even for materials having twinning, small crystallites and possessing a large number of nonequivalent atoms.
${ }^{1}$ F. Walz, J. Phys.: Condens. Matter 14, R285 (2002).

${ }^{2}$ E. J. W. Verwey, Nature (London) 144, 327 (1939); E. J. W. Verwey and P. W. Haayman, Physica (Amsterdam) 8, 979 (1941).

${ }^{3}$ M. Imada, A. Fujimori, and Y. Tokura, Rev. Mod. Phys. 70, 1039 (1998).

${ }^{4}$ M. Coey, Nature (London) 430, 155 (2004).

${ }^{5}$ I. Leonov, A. N. Yaresko, V. N. Antonov, M. A. Korotin, and V. I. Anisimov, Phys. Rev. Lett. 93, 146404 (2004); I. Leonov, A. N. Yaresko, V. N. Antonov, and V. I. Anisimov, Phys. Rev. B 74, 165117 (2006).

${ }^{6}$ Horng-Tay Jeng, G. Y. Guo, and D. J. Huang, Phys. Rev. Lett. 93, 156403 (2004); Phys. Rev. B 74, 195115 (2006).

${ }^{7}$ P. Novák, H. Stepánková, J. Englich, J. Kohout, and V. A. M. Brabers, Phys. Rev. B 61, 1256 (2000).

${ }^{8}$ F. J. Berry, S. Skinner, and M. F. Thomas, J. Phys.: Condens. Matter 10, 215 (1998).

${ }^{9}$ E. Nazarenko, J. E. Lorenzo, Y. Joly, J.-L. Hodeau, D. Mannix, and C. Marin, Phys. Rev. Lett. 97, 056403 (2006).

${ }^{10}$ R. J. Goff, J. P. Wright, J. P. Attfield, and P. G. Radaelli, J. Phys.: Condens. Matter 17, 7633 (2005).

${ }^{11}$ J. García, G. Subías, M. G. Proietti, J. Blasco, H. Renevier, J. L. Hodeau, and Y. Joly, Phys. Rev. B 63, 054110 (2001).

${ }^{12}$ G. Subías, J. García, J. Blasco, M. G. Grazia Proietti, H. Renevier, and M. Concepción Sánchez, Phys. Rev. Lett. 93, 156408 (2004).

${ }^{13}$ J. Schlappa, C. Schüssler-Langeheine, C. F. Chang, H. Ott, A. Tanaka, Z. Hu, M. W. Haverkort, E. Schierle, E. Weschke, G. Kaindl, and L. H. Tjeng, Phys. Rev. Lett. 100, 026406 (2008).

${ }^{14}$ D. J. Huang, H.-J. Lin, J. Okamoto, K. S. Chao, H.-T. Jeng, G. Y. Guo, C.-H. Hsu, C.-M. Huang, D. C. Ling, W. B. Wu, C. S. Yang, and C. T. Chen, Phys. Rev. Lett. 96, 096401 (2006).

${ }^{15}$ G. Kh. Rozenberg, M. P. Pasternak, W. M. Xu, Y. Amiel, M. Hanfland, M. Amboage, R. D. Taylor, and R. Jeanloz, Phys. Rev. Lett. 96, 045705 (2006).

${ }^{16}$ P. Piekarz, K. Parlinski, and A. M. Oleś, Phys. Rev. Lett. 97, 156402 (2006); Phys. Rev. B 76, 165124 (2007).

${ }^{17}$ M. Bimbi, G. Allodi, R. De Renzi, C. Mazzoli, and H. Berger, Phys. Rev. B 77, 045115 (2008).

${ }^{18}$ L. Craco, M. S. Laad, and E. Müller-Hartmann, Phys. Rev. B 74, 064425 (2006).

${ }^{19}$ R. J. McQueeney, M. Yethiraj, S. Chang, W. Montfrooij, T. G. Perring, J. M. Honig, and P. Metcalf, Phys. Rev. Lett. 99, 246401 (2007); 100, 069901(E) (2008); R. J. McQueeney, M. Yethiraj, W. Montfrooij, J. S. Gardner, P. Metcalf, and J. M.
Honig, Phys. Rev. B 73, 174409 (2006).

${ }^{20}$ J. P. Wright, J. P. Attfield, and P. G. Radaelli, Phys. Rev. Lett. 87, 266401 (2001); Phys. Rev. B 66, 214422 (2002).

${ }^{21}$ Y. Miyamoto and M. Shindo, J. Phys. Soc. Jpn. 62, 1423 (1993).

${ }^{22}$ J. M. Bijvoet, Proc. R. Acad. Sci. Amsterdam B52, 313 (1949).

${ }^{23}$ W. A. Hendrickson, Science 254, 51 (1991).

${ }^{24}$ J.-L. Hodeau, V. Favre-Nicolin, S. Bos, H. Renevier, E. Lorenzo, and J.-F. Berar, Chem. Rev. (Washington, D.C.) 101, 1843 (2001).

${ }^{25}$ Y. Murakami, H. Kawada, H. Kawata, M. Tanaka, T. Arima, Y. Moritomo, and Y. Tokura, Phys. Rev. Lett. 80, 1932 (1998).

${ }^{26}$ Y. Joly, S. Grenier, and J. E. Lorenzo, Phys. Rev. B 68, 104412 (2003).

${ }^{27}$ M. v. Zimmermann, C. S. Nelson, J. P. Hill, Doon Gibbs, M. Blume, D. Casa, B. Keimer, Y. Murakami, C.-C. Kao, C. Venkataraman, T. Gog, Y. Tomioka, and Y. Tokura, Phys. Rev. B 64, 195133 (2001)

${ }^{28}$ S. B. Wilkins, P. D. Spencer, P. D. Hatton, S. P. Collins, M. D. Roper, D. Prabhakaran, and A. T. Boothroyd, Phys. Rev. Lett. 91, 167205 (2003).

${ }^{29}$ Doon Gibbs, D. R. Harshman, E. D. Isaacs, D. B. McWhan, D. Mills, and C. Vettier, Phys. Rev. Lett. 61, 1241 (1988).

${ }^{30}$ D. H. Templeton and L. K. Templeton, Acta Crystallogr., Sect. A: Found. Crystallogr. 41, 365 (1985).

${ }^{31}$ V. E. Dmitrienko, Acta Crystallogr., Sect. A: Found. Crystallogr. 39, 29 (1983).

${ }^{32}$ M. Blume, in Resonant Anomalous X-Ray Scattering, edited by G. Materlik, J. Sparks, and K. Fisher (Elsevier, Amsterdam, 1994), p. 495.

${ }^{33}$ S. Di Matteo, Y. Joly, and C. R. Natoli, Phys. Rev. B 72, 144406 (2005).

${ }^{34}$ S. Grenier, A. Toader, J. E. Lorenzo, Y. Joly, B. Grenier, S. Ravy, L. P. Regnault, H. Renevier, J. Y. Henry, J. Jegoudez, and A. Revcolevschi, Phys. Rev. B 65, 180101(R) (2002).

${ }^{35}$ S. Fagot, P. Foury-Leylekian, S. Ravy, J.-P. Pouget, E. Lorenzo, Y. Joly, M. Greenblatt, M. V. Lobanov, and G. Popov, Phys. Rev. B 73, 033102 (2006).

${ }^{36} \mathrm{~J}$. Philip and J. Rundgren, in Proceedings of the Conference on Determination of Surface Structure by LEED, Yorktown Heights, 1980, edited by P. M. Marcus (Plenum, New York, 1984).

${ }^{37}$ T. N. Horsky, G. R. Brandes, K. F. Canter, C. B. Duke, A. Paton, D. L. Lessor, A. Kahn, S. F. Horng, K. Stevens, K. Stiles, and A. P. Mills, Phys. Rev. B 46, 7011 (1992). 
${ }^{38}$ Y. Joly, Phys. Rev. B 63, 125120 (2001); The code can be freely downloaded at the web address www.neel.cnrs.fr/fdmnes

${ }^{39}$ L. Hedin and S. Lundqvist, J. Phys. C 4, 2064 (1971).

${ }^{40}$ C. Medrano, M. Schlenker, J. Baruchel, J. Espeso, and Y. Miya- moto, Phys. Rev. B 59, 1185 (1999).

${ }^{41}$ F. Delille, B. Dieny, J.-B. Moussy, M.-J. Guittet, S. Gota, M. Gautier-Soyer, and C. Marin, J. Magn. Magn. Mater. 294, 27 (2005). 